\title{
GAMMA-RAY EMISSION OF ACTIVE GALACTIC NUCLEI AS A SIGNATURE OF RELATIVISTIC ELECTRON-POSITRON BEAMS
}

\author{
G. Henri and G. Pelletier \\ Laboratoire d'Astrophysique, Observatoire de Grenoble, Université Joseph Fourier, BP 53X, F38041 Grenoble, France \\ AND \\ J. RoLAND \\ Institut d'Astrophysique, 98 bis, Boulevard Arago, F75014 Paris, France; and \\ Leiden Observatory, The Netherlands \\ Received 1992 June I; accepted 1992 November 20
}

\begin{abstract}
Gamma-ray emission from extragalactic sources is interpreted as the Doppler-boosted annihilation and inverse Compton radiation from a relativistic electron-positron beam. We argue that Doppler boosting is required to avoid a strong depletion by $\gamma-\gamma$ pair production, but even so, the compactness cannot be smaller than unity at light-week scale with a reasonable Doppler factor. This strongly suggests the existence of a beamed electron-positron source. The case of the quasar 3C 279 is investigated in detail. We show that one can easily account for the observed luminosity and variability time scale with reasonable values of the plasma density and bulk Lorentz factor of the beam, in accordance with previous estimates based upon observations at other wavelengths (radio and X-rays). The observations put new constraints on the size, the geometry, and the particle distribution function of the source. We discuss briefly the link between $\gamma$-ray and radio VLBI observations.
\end{abstract}

Subject headings: galaxies: jets — gamma rays: theory - quasars: general

\section{INTRODUCTION}

Compton Gamma Ray Observatory (CGRO) observations have revealed intense $\gamma$-ray emission from several active galactic nuclei and quasars (see $\S 2$ ). Many processes for $\gamma$-radiation production have been proposed: inverse Compton (IC) effect, hadronic processes through relativistic neutrons, Penrose quantum effect, and emission from an optically thin accretion disk at very high temperature. Except for the inverse Compton effect from a relativistic jet, none of these processes appears to fulfill the constraints on the observed luminosity, variability, and spectrum (Hartman et al. 1992c and references therein). Indeed, the very high intensity seems to imply an anisotropic emission, such as that invoked to explain the intense radio emission of these objects. Furthermore, in the latter case, a Doppler boosting by a relativistic jet is necessary to avoid the inverse Compton catastrophe by reducing the apparent luminosity to much lower values. A similar constraint arises for $\gamma$-radiation, since a high photon density would produce intense pair production and thus completely deplete the source. However, we will show in $\S 3.3$ that a reasonable value of bulk Lorentz factor together with the variability maximum size imply a compactness of order unity, suggesting the existence of copious pair production in a more compact region. Hence we propose to attribute $\gamma$-ray and radio emissions to the same $e^{-}-e^{+}$plasma ejected relativistically. In our model, the $\gamma$-ray emission is produced by the IC process in the high-energy tail (EGRET hard $\gamma$-ray spectrum), whereas the COMPTEL measurements contain an $e^{-}-e^{+}$annihilation component. The emitting region corresponds to the decompaction of the beam at few light-days from the nucleus, and the radio emission is a synchrotron emission produced after a few light-weeks from the nucleus. This appears as a natural consequence of the model of electron-positron beam formation proposed recently (Henri \& Pelletier 1991).
Since the $\gamma$-ray observations already published concern $3 \mathrm{C} 279$, we will focus our discussion on this source to deduce constraints on the relativistic $e^{-}-e^{+}$beam taking into account VLBI observations.

\section{PRESENT STATUS OF GAMMA-RAY OBSERVATIONS OF ACTIVE GALACTIC NUCLEI}

\subsection{CGRO Observations}

Since the launch of the GRO satellite, 14 extragalactic radio sources have been detected as $\gamma$-ray sources before 1992 May 20 (Table 1). All of them contain a compact radio source associated with the nucleus of the galaxy. The flux densities of the compact sources are greater than $1 \mathrm{Jy}$ at $5 \mathrm{GHz}$, and their radio spectral indices $\alpha_{r}$ are smaller than 0.5 [the radio spectral index is defined as (flux density) $\propto$ (frequency) $^{-\alpha_{r}}$. Five of them (PKS 0235 + 164, 4C 71.07, 3C 273, 3C 279, and 3C 454.3) are well-known superluminal radio sources, and two (Mrk 421 and CTA 102) are probably superluminal; the others do not have different VLBI observation epochs (see Zensus \& Pearson 1987, 1989 for VLBI radio observations).

The radio properties of these galaxies, i.e., flux density and structure variability, and their large flux densities at $5 \mathrm{GHz}$, suggest an anisotropic synchrotron emission, i.e., a Doppler beaming amplification due to a relativistic ejection of the $e^{-}$and $e^{+}$responsible for the VLBI synchrotron emission. The high level of their $\gamma$-ray emission suggests that their $\gamma$-ray emission is also anisotropic (see Hartman et al. 1992c and Hermsen et al. 1992 in the case of 3C 279), i.e., the plasma responsible for the $\gamma$-ray emission is also moving relativistically.

\subsection{The $\gamma$-Ray Spectrum of $3 C 279$}

Recent $\gamma$-ray observations of 3C 279 have been reported by Hartman et al. (1992c) and Hermsen et al. (1992). The $\gamma$-ray spectrum is shown in Figure 1. This spectrum exhibits a 
TABLE 1

Extragalactic Gamma-Ray Sources

\begin{tabular}{|c|c|c|c|c|c|c|c|}
\hline \multirow[b]{2}{*}{ NAME } & \multicolumn{2}{|c|}{ Position } & \multirow[b]{2}{*}{ IDENTIFICATION } & \multirow[b]{2}{*}{$z$} & \multirow[b]{2}{*}{$S(5 \mathrm{GHz})$} & \multirow[b]{2}{*}{$\alpha_{r}$} & \multirow[b]{2}{*}{ REFERENCE } \\
\hline & $\alpha$ & $\delta$ & & & & & \\
\hline PKS 0202+149 ...... & $02^{\mathrm{h}} 02^{\mathrm{m}}$ & $+14: 9$ & EF & & $\simeq 2 \mathrm{Jy}$ & $\leq 0.5$ & 1 \\
\hline PKS 0208-512 ....... & 0208 & -51.2 & $\mathbf{Q}$ & 1.003 & $\simeq 3 \mathrm{Jy}$ & 0.2 & 2 \\
\hline PKS $0235+164 \ldots \ldots$. & 0235 & +16.4 & BL & 0.940 & $\simeq 2 \mathrm{Jy}$ & -0.5 & 1 \\
\hline PKS 0420-014 ....... & 0420 & -01.4 & Q & 0.915 & $\simeq 1.5 \mathrm{Jy}$ & $\leq 0$ & 1 \\
\hline PKS $0528+134 \ldots \ldots \ldots$ & 0528 & +13.4 & $\dot{Q}$ & & $\simeq 4 \mathrm{Jy}$ & $\leq 0$ & 2 \\
\hline PKS 0537-441 ....... & 0537 & -44.1 & $\mathbf{Q}$ & 0.894 & $\simeq 4 \mathrm{Jy}$ & -0.1 & 3 \\
\hline S5 $0716+71 \ldots \ldots \ldots \ldots$ & 0716 & +71.4 & BL? & & $\simeq 1 \mathrm{Jy}$ & -0.2 & 3 \\
\hline $4 \mathrm{C} 71.07 \ldots \ldots \ldots \ldots$ & 0836 & +71.0 & Q & 2.16 & $\simeq 2 \mathrm{Jy}$ & 0.3 & 4 \\
\hline Mrk 421 .............. & 1101 & +38.4 & BL & 0.031 & $\simeq 0.7 \mathrm{Jy}$ & -0.3 & 3 \\
\hline $3 C 273 \ldots \ldots \ldots \ldots \ldots$ & 1226 & +02.3 & Q & 0.158 & $\simeq 40 \mathrm{Jy}$ & -0.1 & 5 \\
\hline 3С 279 ................ & 1253 & -05.5 & $\mathbf{Q}$ & 0.536 & $\simeq 15 \mathrm{Jy}$ & -0.4 & 6 \\
\hline 4C $38.41 \ldots \ldots \ldots \ldots \ldots$ & 1633 & +38.2 & $\mathrm{Q}$ & 1.814 & $\simeq 4 \mathrm{Jy}$ & -0.8 & 2 \\
\hline CTA $102 \ldots \ldots \ldots \ldots \ldots$ & 2230 & +11.4 & $\mathrm{Q}$ & 1.037 & $\simeq 3.7 \mathrm{Jy}$ & 0.5 & 7 \\
\hline $3 \mathrm{C} 454.3 \ldots \ldots \ldots \ldots$ & 2251 & +15.8 & $\mathbf{Q}$ & 0.859 & $\simeq 10 \mathrm{Jy}$ & 0.1 & 7 \\
\hline
\end{tabular}

REFERENCES.-Positions, identifications, redshifts, flux densities at $5 \mathrm{GHz}$, and spectral indices calculated between 2.7 and $5 \mathrm{GHz}$ can be found in Véron-Cetty \& Véron 1991 and in Kühr et al. 1979 in the case of PKS $0202+149$ and PKS 0528 + 134. Gamma-ray observations and identifications are reported by the following: (1) Hartman et al. $1992 \mathrm{~b}$. (2) Kanbach et al. 1992. (3) Michelson et al. 1992; Weekes 1992. (4) Fichtel et al. 1992. (5) Hermsen et al. 1992. (6) Bertsch et al. 1991; Kanbach et al. 1992; Hartman et al. 1992c; Hermsen et al. 1992. (7) Hartman et al. 1992a.

maximum emission per logarithmic energy interval of $\sim 10$ $\mathrm{MeV}$ and a break of photon spectral index from approximately 1.5 to 2 .

\section{INTERPRETATION OF THE 3C 279 GAMMA-RAY EMISSION}

\subsection{The Two-Flow Model of Extragalactic Jets}

The $\gamma$-ray emission is a natural consequence of the two-flow model that has been previously proposed to understand extragalactic radio jets. Indeed, the general properties of extragalactic radio sources can be understood if their nuclei are ejecting two fluids (Sol, Pelletier, \& Asséo 1989; Pelletier, Sol, \& Asséo 1988; Roland, Lehoucq, \& Pelletier 1992):

1. A relativistic $e^{-}-e^{+}$beam with speed $v_{b} \simeq c$. Its bulk Lorentz factor is $3 \leq \gamma_{b} \leq 20$, and it is responsible for the formation of superluminal radio sources.

2. A nonrelativistic $e^{-}-p$ jet with speed $v_{j} \simeq 0.4 c$ and a mass ejection rate $\dot{M}_{j} \leq 0.5 \times M_{\odot} \mathrm{yr}^{-1}$. It is responsible for the formation of hot spots and extended lobes. It contains most of the mass and the kinetic power of the material ejected by the nuclei.

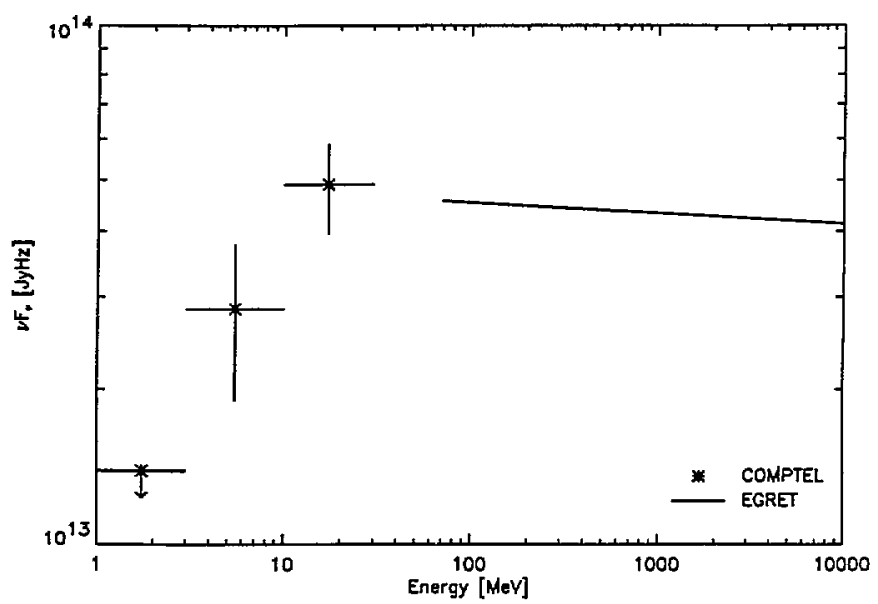

FIG. 1.-Gamma-ray spectrum of 3C 279 obtained by COMPTEL and EGRET and published by Hermsen et al. (1992).
In the first light-hours, the compactness is high enough to make the plasma opaque to $\gamma$-photons, owing to $\gamma-\gamma$ pair production. Pair annihilation is thus negligible, since most $\gamma$-photons produced by an annihilation event will be eventually reabsorbed and create a new pair. Between 1 light-day and $\sim 1$ light-week, the accelerated beam becomes transparent to $\gamma$ photons because of its transversal expansion, but the radius of the beam is still small enough to allow $e^{-}-e^{+}$annihilations to take place. This ceases when most low-energy pairs have annihilated, defining the $\gamma$-ray emission region. The beam then becomes transparent in the millimeter range after a few lightweeks, producing VLBI components by synchrotron emission from highly relativistic pairs.

\subsection{Integrated Luminosity}

For an unresolved source, the apparent flux density, $S_{\text {ap }}(v)$, is related to the intrinsic flux density, $S(v)$, by

$$
S_{\mathrm{ap}}(v)=\delta^{3} S\left(v^{\prime}\right),
$$

where $\delta \equiv \gamma_{b}\left(1-\beta_{b} \cos \theta\right)^{-1}$ is the Doppler factor. The observed frequency $v$ results from blueshifting the emitted frequency $v^{\prime}$ by a factor $\delta$. For small viewing angle $\theta$ and large Lorentz factor $\gamma_{b}$, we get the approximate value of the Doppler factor $\delta \simeq 2 \gamma_{b} /\left(1+\theta^{2} \gamma_{b}^{2}\right)$.

Thus, the apparent integrated luminosity $L_{\gamma \text {, ap }}$ is related to the intrinsic integrated luminosity $L_{\gamma}$ by

$$
L_{\gamma, \text { ap }}=\delta^{4} L_{\gamma} \text {. }
$$

The observed $\gamma$-ray spectrum of 3C 279 between $100 \mathrm{MeV}$ and $10 \mathrm{GeV}$ is (Hartman et al. 1992c)

$$
d J / d \epsilon \simeq 2.7 \times 10^{-7} \epsilon^{-2} \text { photons } \mathrm{cm}^{-2} \mathrm{~s}^{-1} \mathrm{GeV}^{-1} \text {. }
$$

The redshift of $3 \mathrm{C} 279$ is $z \simeq 0.536$, so assuming for the Hubble constant $H_{0} \simeq 100 \mathrm{~km} \mathrm{~s}^{-1} \mathrm{Mpc}^{-1}$ and $q_{0} \simeq 0.5$, the luminosity distance of $3 \mathrm{C} 279$ is $D_{L} \simeq 2900 \mathrm{Mpc}$. The apparent integrated luminosity of $3 \mathrm{C} 279$ between $100 \mathrm{MeV}$ and $10 \mathrm{GeV}$ is

$$
L_{\gamma, \mathrm{ap}} \simeq 4 \pi D_{L}^{2} \int \epsilon(d J / d \epsilon) d \epsilon \simeq 2 \times 10^{48} \mathrm{ergs} \mathrm{s}^{-1}
$$


Superluminal motion has been detected for VLBI components of $3 \mathrm{C} 279$, and the Doppler factor $\delta$ of the beam can be of order 10. Thus the annihilation spectrum will begin for energies greater than $5 \mathrm{MeV}$. This shape of the spectrum corresponds to the $\gamma$-ray spectrum observed by COMPTEL (Hermsen et al. 1992) and shown in Figure 1. With this value of $\delta$, the intrinsic $\gamma$-ray luminosity between $10 \mathrm{MeV}$ and $1 \mathrm{GeV}$ is $L_{\gamma} \simeq 2 \times 10^{44}$ ergs s $^{-1}$.

This value of the $\gamma$-ray luminosity corresponds to a highactivity phase; the observations indicate variability by a factor of at least 5; so, in the quiet phase, the luminosity is about a few times $10 \%$ of the previous value: $L_{\gamma} \simeq 4 \times 10^{43} \mathrm{ergs} \mathrm{s}^{-1}$.

\subsection{Geometrical Constraints}

Observation of copious $\gamma$-ray emission near the pairproduction threshold implies that this process occurs beyond the region of high compactness to avoid strong depletion by photon-photon pair production. This puts a constraint on the size of this emitting region. We assume that this region is a portion of a cylinder of radius $r_{b}$ and length $h$ in the observer frame, in which the plasma has a bulk Lorentz factor $\gamma_{b}$. In the plasma frame, this region has a lateral area $S_{b}=2 \pi r_{b} h \gamma_{b}^{-1}$ and a volume $V_{b}=\pi r_{b}^{2} h \gamma_{b}^{-1}$. Let us define the compactness $l$ as the optical depth of the population photons with energies of a few $\mathrm{MeV}$ with respect to photon-photon pair production:

$$
l=\sigma_{\mathrm{T}} r_{b} n(\epsilon \simeq 1)
$$

where the plasma frame photon density per unit energy $n(\epsilon \simeq 1) \simeq L_{\gamma}^{<} / m_{e} c^{3} S_{b} \Delta \epsilon, L_{\gamma}^{<}$being the plasma frame luminosity of photons with energies of a few MeV in the interval $\Delta \epsilon$. This leads to a compactness that depends on the length of the emitting region but not on its radius:

$$
l=\frac{L_{\gamma}^{<} \sigma_{\mathrm{T}} \gamma_{b}}{2 \pi h m_{e} c^{3} \Delta \epsilon} .
$$

To get a compactness smaller than unity, one needs a lower limit for the length $h$ :

$$
h \simeq \frac{L_{\gamma}^{<} \sigma_{\mathrm{T}} \gamma_{b}}{2 \pi m_{e} c^{3} \Delta \epsilon} \simeq 1.6\left(\frac{L_{\gamma, \mathrm{ap}}^{<}}{4 \times 10^{47}}\right)\left(\frac{\delta}{5}\right)^{-3}\left(\frac{\gamma_{b}}{10}\right) \text { light-days }
$$

where the observed luminosity $L_{\gamma \text {, ap }}^{<}$in the $3-30 \mathrm{MeV}$ range is about $4 \times 10^{47}$ ergs s$^{-1}$ (Hermsen et al. 1992) (note that this range corresponds to photons with energies of a few $\mathrm{MeV}$ in the rest frame, with $\Delta \epsilon \simeq 30 / \delta$ ). For $\delta=5$ and $\gamma_{b}=10$, one gets a length of a few light-days, which is compatible with the currently observed variability time scale lower than 1 week. It is important to note that the raw $\gamma$-ray luminosity would give a much higher value, of the order of some light-years. So it would be very difficult to account for the variability without a Doppler boosting and beaming by a relativistic beam. Alternatively, a compactness much lower than unity would require either a larger Doppler factor, which would exceed the upper bound set up by VLBI observations, or a larger size, which would exceed the upper bound set up by the variability time scale.

This time scale can be interpreted as the annihilation time in the observer frame (see next section); for the sake of simplicity, let us adopt the following approximation for the pair annihilation rate between an isotropic population of electrons and positrons with Lorentz factors $\gamma_{-}$and $\gamma_{+}$(Coppi \& Blandford 1990):

$$
R_{ \pm}=\frac{3}{8} \frac{c \sigma_{\mathrm{T}}}{x}\left(\ln x+x^{-1 / 2}\right)
$$

where $x=\gamma_{-} \gamma_{+}$. We thus deduce an estimate of the lowenergy pair density in the plasma frame:

$$
n_{ \pm} \simeq \frac{8 \gamma_{b}}{3 h \sigma_{\mathrm{T}}} \simeq 10^{9}\left(\frac{\gamma_{b}}{10}\right)\left(\frac{1}{h \text { light-days }}\right) \mathrm{cm}^{-3} .
$$

This density can be easily achieved in the relativistic beam model in which a pair-creation catastrophe takes place in the source because of in situ turbulent acceleration by Alfven waves (Henri \& Pelletier 1991): in this model, the expected energy mass density is of the order of the magnetic density. For instance, a pair density of $10^{9}$ would correspond to a magnetic field $B \sim 100 \mathrm{G}$, in agreement with the estimate that one gets from a magnetic field of $10 \mathrm{kG}$ at a compact scale of 1 lighthour. The synchrotron loss time is very short; however, the source is still optically thick to synchrotron radiation, and thus the energy loss is balanced by reabsorption. Moreover, it has been shown that in situ reacceleration by Alfven waves in the compact source can really be efficient to balance radiation loss, especially the important inverse Compton radiation. Furthermore, as long as the beams travels away from the nucleus, the inverse Compton loss weakens more rapidly than the in situ acceleration, so the average energy of the pairs can easily increase up to $\mathrm{GeV}$ energy at light-week distance from the nucleus.

Now the total intrinsic luminosity due to low-energy annihilation is

$$
L_{\mathrm{ann}} \simeq\left(\frac{3}{8} \sigma_{\mathrm{T}} n_{ \pm}^{2} c\right)\left(\pi r_{b}^{2} h \gamma_{b}^{-1}\right)\left(m_{e} c^{2}\right)
$$

Combining equations (7), (9), and (10), and noting that $L_{\text {ann }}<$ $L_{\gamma}^{<}$, one gets an upper limit of the radius of the beam:

$$
r_{b}<\gamma_{b}^{-1} h
$$

that is, the emitting region must be a rather thin beam with an aspect ratio $\rho_{a} \equiv r_{b} / h<\gamma_{b}^{-1}$. Conversely, it could appear nearly spherical in the plasma frame. The creation rate $\dot{M}_{ \pm}$is $\simeq 1.2 \times 10^{-3} M_{\odot} \mathrm{yr}^{-1}$. This clearly shows that the relativistic ejection is weak and that the large-scale jet cannot be due to the relativistic flow.

\subsection{The $\gamma$-Ray Spectrum}

We consider now the high-energy power-law $\gamma$-ray spectrum observed between $100 \mathrm{MeV}$ and $10 \mathrm{GeV}$. At high energy, one can show that the IC process, considered by other authors (Dermer, Schlickeiser, \& Mastichiadis 1992; Maraschi, Ghisellini, \& Celotti 1992), necessarily dominates over annihilation. Indeed, the photon production rates at an energy $\epsilon m_{e} c^{2}$ due to annihilation and IC process are respectively

$$
\dot{n}_{\mathrm{ann}}(\epsilon)=\frac{3 c \sigma_{\mathrm{T}} n_{<} n(\epsilon)}{8 \epsilon} \text { and } \dot{n}_{\mathrm{IC}}(\epsilon)=\frac{c \sigma_{\mathrm{T}} n_{s} n(\gamma)}{\gamma \epsilon_{s}}
$$

where $n_{s}$ and $\epsilon_{s}$ are respectively the spatial density and the mean energy in units $m_{e} c^{2}$ of the soft photons, and $\gamma=$ $\left(3 \epsilon / 4 \epsilon_{s}\right)^{1 / 2}$. Assuming a power-law energy distribution of relativistic particles, $n(\gamma) \propto \gamma^{-p}$, the ratio of the two rates is given by

$$
\frac{\dot{n}_{\mathrm{IC}}(\epsilon)}{\dot{n}_{\mathrm{ann}}(\epsilon)}=\frac{8 n_{s}}{3 n_{<} \epsilon_{\mathrm{s}}}\left(\frac{\gamma}{\epsilon}\right)^{-(p+1)} \simeq \frac{n_{\mathrm{s}}}{n_{<} \epsilon_{s}}\left(\epsilon \epsilon_{s}\right)^{(p+1) / 2} .
$$

Taking $\epsilon_{s}=10^{-4}$ and $L_{s}=10^{46} \mathrm{ergs} \mathrm{s}^{-1}$, one finds that the IC process dominates above $\epsilon \sim 10$ for $p=3$. Note that annihilation can actually be important at lower energies.

For an optically thin source, a $\gamma$-ray spectral index of unity (in energy) would be produced by a power-law distribution of 
electron Lorentz factors with an index $p=3$. This can be obtained in the downstream flow of a strong shock by the radiation cooling of a $\gamma^{-2}$ distribution. However, opacity effects can modify this value: indeed, if the region is marginally thin for photons with $\epsilon \sim 1$, one would expect it to be optically thick to higher energy photons, which interact predominantly with X-photons of energy $(1 / \epsilon) m_{e} c^{2}$ with an optical depth $\tau_{y \gamma} \sim[n(1 / \epsilon) / \epsilon] \sigma_{T} r_{b}$. In the latter case, the observed $\gamma$-ray spectral index is $\alpha_{\gamma}=2 \alpha_{X}$ (Svensson 1984). The observed value $\alpha_{\gamma} \simeq 1$ should correspond to $\alpha_{X}=0.5$, close to that observed, and would be produced by a distribution of index $p=2$. Such a power law could be due to the Compton cooling of a monoenergetic injected distribution of pairs.

In all cases, the high-energy tail could be rapidly set up by the acceleration of the low-energy pair plasma. Flares like that detected by EGRET could be due to an increase of pair density followed by a subsequent annihilation. If the acceleration time is much shorter than the annihilation time, the shape of the distribution will remain constant while the intensity changes on a time scale governed by the slow annihilation process.

Recently, the Whipple Observatory has detected $\mathrm{TeV}$ photons from the object Mrk 421, corresponding to $\epsilon=10^{6}$, at a level close to the value extrapolated from EGRET measurements; such a high value seems to lie above the Klein-Nishina regime: this should imply first a much lower production rate, and second a stronger absorption by direct pair production with UV photons; however, taking into account a bulk Lorentz factor of 10 , the plasma frame energy of the $\mathrm{TeV}$ photons is reduced to $10^{5}$, whereas that of UV photons is of the order of $10^{-8}$ if they are beamed along the axis (which is expected if they come from an accretion disk (Dermer et al. 1992) or from the relativistic jet itself): Whipple results could then correspond to the extreme limit of the Thomson regime for an energy distribution extending up to $\gamma=10^{5}$.

\section{CONCLUSION}

The discovery of powerful $\gamma$-ray sources associated with compact and variable extragalactic radio sources, whose VLBI radio emission is anisotropic, suggests that both the $\gamma$-ray and the radio emissions are anisotropic and occur in the same plasma ejected relativistically by the nucleus of the galaxy. The $\gamma$-ray emission of extragalactic radio sources can be explained by the radiation of the relativistic $e^{+}$and $e^{-}$of the beam. It is a natural consequence of the two-flow model when the compact source becomes transparent to $\gamma$-rays, which corresponds to the annihilation zone. The required pair density can be easily obtained by a turbulent pair-creation catastrophe model.

In the case of $3 \mathrm{C} 279$, one can account for the observed $\gamma$-ray spectrum (Hermsen et al. 1992) with a bulk Lorentz factor $\gamma_{b} \simeq 10$, an angle between the line of sight and the ejection direction $\theta \simeq 8^{\circ}$, and a resulting blueshifted Doppler factor $\delta \simeq 5$. These values agree with those inferred from the lack of inverse Compton catastrophe (see Unwin et al. 1989). The mass ejected in the beam must be $\dot{M}_{ \pm} \leq 10^{-3} M_{\odot} \mathrm{yr}^{-1}$.

For 3C 279 the annihilations occur in the beam during the first light-weeks. After 1 light-week, most of the low-energy $e^{-}$ and $e^{+}$have annihilated to produce the $\gamma$-ray emission. In the case of $3 \mathrm{C} 273$, VLBI observations indicate than the mass ejected in the beam is $\dot{M}_{ \pm} \leq 10^{-5} M_{\odot} \mathrm{yr}^{-1}$ (Pelletier \& Roland 1989), which is compatible with the previous values if $1 \%$ of the pairs escape from the annihilation region.

Among the 14 sources detected by CGRO, two $(0836+710$ and Mrk 421) have shown an optical burst in the same time as the $\gamma$-ray observation (Schramm et al. 1992; Hurst 1992; Toone \& Worraker 1992; Fiorucci \& Tosti 1992). As indicated for $3 \mathrm{C} 279$, the synchrotron radiation of $e^{-}$and $e^{+}$with energies of a few $\mathrm{GeV}$ in the beam occur at frequencies greater than $10^{13} \mathrm{~Hz}$, i.e., in the optical range. Thus the observed optical burst can be the optical synchrotron radiation due to the beam.

After $\sim 2$ light-weeks the beam becomes transparent to millimeter radiations, so it will be of the greatest interest to see whether a $\gamma$-ray burst similar to that observed in 1991 for 3C 279 is followed by a millimeter burst after a few weeks or a few days.

One of us (J. R.) acknowledges fruitful discussions with W. Hermsen and thanks him for his useful comments. We appreciated the constructive comments of an anonymous referee.
Bertsch, D. L., et al. 1991, IAU Circ., No. 5311

Coppi, P. S. \& Blandford, R. D. 1990, MNRAS, 245, 453

Dermer, C. D., Schlickeiser, R., \& Mastichiadis, A. 1992, A\&A, 256, L27

Fichtel, C. E., et al. 1992, IAU Circ., No. 5460

Fiorucci, M., \& Tosti, G. 1992, IAU Circ., No. 5496

Hartman, R. C., et al. 1992a, IAU Circ., No. 5477

Hartman, R. C., et al. 1992b, IAU Circ., No. 5519

Hartman, R. C. et al. 1992c, ApJ, 385, L1

Henri, G., \& Pelletier, G. 1991, ApJ, 383, L7

Hermsen, W., et al. 1992, A\&AS, in press

Hurst, G. M. 1992, IAU Circ., No. 5471

Kanbach, G., et al. 1992, IAU Circ., No. 5431

Kühr, H., Nauber, U., Pauliny-Toth, I. I. K., \& Witzel, A. 1979, A Catalogue of Radio Sources, A\&AS, 45, 367

Maraschi, L., Ghisellini, G., \& Celotti, A. 1992, in The Nature of Compact Objects in AGN (Cambridge: Cambridge Univ. Press), in press

Michelson, P. F., et al. 1992, IAU Circ., No. 5470.

Pelletier, G., Sol, H., \& Asséo, E. 1988, Phys. Rev. A, 38, 2552

\section{REFERENCES}

Pelletier, G., \& Roland, J. 1989, A\&A, 224, 24

Roland, J., Lehoucq, R., \& Pelletier, G. 1992, in Extragalactic Radio Sources: From Beams to Jets, ed. J. Roland, H. Sol, \& G. Pelletier (Cambridge: Cambridge Univ. Press)

Schramm, J., Linde, J. V., Borgeest, U., \& Mieser, L. 1992, IAU Circ., No. 5453

Sol, H., Pelletier, G., \& Asséo, E. 1989, MNRAS, 237, 411

Svensson, R. 1984, in X-Ray Emission from Active Galactic Nuclei, ed. J. Trümper \& W. Brinkmann (Garching: MPI)

Toone, J., \& Worraker, B. 1992, IAU Circ., No. 5471

Unwin, S. C., Cohen, M. H., Biretta, J. A., Hodges, M. W., \& Zensus, J. A. 1989, ApJ, 340, 117

Véron-Cetty, M. P. \& Véron, P. 1991, A Catalogue of Quasars and Active Nuclei: Fifth Edition (ESO Sci. Rep., No. 10)

Weekes, T. C. 1992, IAU Circ., No. 5522

Zensus, J. A., \& Pearson, T. J., eds. 1987, Superluminal Radio Sources (Cambridge: Cambridge Univ. Press)

. 1989, Parsec-Scale Radio Jets (Cambridge: Cambridge Univ. Press) 